\title{
Can the diagnosis of recurrent vulvovaginal candidosis be improved by use of vaginal lavage samples and cultures on chromogenic agar?
}

\author{
N. Novikova ${ }^{1}$, A. Rodrigues ${ }^{2}$ and P.-A. Mårdh ${ }^{1}$ \\ ${ }^{1}$ Department of Obstetrics and Gynecology, Lund University Hospital, Lund, Sweden \\ ${ }^{2}$ Department of Microbiology, Porto Medical School, Porto, Portugal
}

\begin{abstract}
Objective: To investigate if introital and vaginal flushing samples inoculated on chromogenic agar could increase the recovery rate and rapid identification of Candida and non-albicans species, as compared to culture of posterior vaginal fornix samples on Sabouraud agar and speciation of isolates by biochemical tests.

Methods: Samples from the introitus and the posterior vaginal fornix and vaginal lavage samples were collected from 91 women with a history suggestive of recurrent vulvovaginal candidosis (RVVC), and with a suspected new attack of the condition. The specimens were cultured on Sabouraud and CHROMagar ${ }^{\circledR}$. Speciation of yeast isolates was made on the chromogenic agar by API $32 \mathrm{C}^{\circledR}$ kits and by an atomized system $\left(\right.$ Vitek $\left.^{\circledR}\right)$.

Results: Forty-six (5 I\%) women were positive for Candida from one or more of the samples. The introital cultures were positive in 43 (47\%) women, both on Sabouraud and chromogenic agar. From the posterior vaginal fomix, $42(46 \%)$ women were positive on the Sabouraud and $43(47 \%)$ on chromogenic agar cultures, while the vaginal lavage cultures yielded Candida on those two media in 40 (44\%) and $4 \mathrm{I}(45 \%)$ cases, respectively. Candida albicans was the most frequent species recovered, from 40 (87\%) cases, followed by $C$. krusei in $4(9 \%)$, C. glabrata in 2 (4\%), and $C$. parapsilosis in one case. There was only one woman who had a mixed yeast infection, by $C$. albicans and $C$. krusei. There was only one discrepancy in the speciation as demonstrated by mean of chromogenic agar and API $32 C$ kit.

Conclusions: Neither cultures of introital nor of vaginal lavage samples increases the detection rate of Candida in RVVC cases as compared to cultures of posterior vaginal fornix samples. Use of chromogenic agar is a convenient and reliable means to detect colonization by Candida and differentiate between C. albicans and non-albicans species.
\end{abstract}

Key words: Vulvovaginal Candidosis; Candida albicans; Non-Albicans Species; Vaginal Lavage; CHromogenic Agar

The most common means to diagnose vulvovaginal Candida infections is to study a wet smear of vaginal secretion that has been treated by potassium hydroxide (KOH) for Candida morphotypes, and/or to culture samples collected with a cotton-tipped swab from the posterior vaginal fornix. The specimens can either be sent to a laboratory for culture and speciation of Candida isolates or plated on Sabouraud agar directly at the clinic $^{1-3}$.

Although the vast majority of strains isolated from the genital tract in recurrent vulvovaginal

Correspondence to: Per-Anders Mårdh, MD, PhD, Department of Obstetrics and Gynecology, University Hospital, SE-221 85 Lund, Sweden. E-mail: per-anders.mardh@simrishamn.se 
candidosis (RVVC) cases belongs to C. albicans ${ }^{4}$, it may be essential to identify instances of colonization with non-albicans species. Non-albicans strains have a natural greater resistance to the antifungal drugs most commonly used to treat vulvovaginal candidosis (VVC), i.e. various azole drugs ${ }^{5,6}$. By the use of chromogenic agar ${ }^{7,8}$ it is possible to directly identify a number of non-albicans species of Candida by mere inspection of the plates, i.e. C. albicans, C. tropicalis, and C. krusei, according to the package insert ${ }^{7}$. Koehler and co-workers ${ }^{9}$ reported, however, that as many as eight Candida species could be identified on chromogenic agar. The species identities on chromogenic agar correspond well to those obtained by fermentation and assimilation tests ${ }^{10}$. Apart from use of samples collected by cotton-tipped swabs, analyses of vaginal flushing samples have been proved of value in the diagnosis of genital pathogens ${ }^{11}$.

A large proportion of women suspected on the basis of history and clinical examination prove negative for Candida on culture of vaginal posterior fornix samples. One hypothesis is that analysis of vaginal lavage samples would increase the chance of detecting only a low number of Candida organisms in the vagina compared to the most commonly applied sampling technique, which involves just touching the vaginal mucosa with a swab. This latter procedure may not leave enough time for adsorption of yeast organisms by the swab. Even if rubbing the mucosa, many organisms may remain attached to the swab material after inoculation of culture media, resulting in a negative culture.

The present study explored the possibility of increasing the detection rate of Candida in women with a history suggestive of RVVC and who did attend with symptoms and signs that were consistent with a new attack of the condition. Analyses of introital swab and vaginal lavage samples were made. Furthermore, the value of chromogenic agar for culture of such specimens was investigated.

\section{SUBJECTS AND METHODS}

The 91 women recruited to the study had all attended the outpatient department of the Maternity Hospital 3, Kiev, because of genital symptoms and a history suggestive of RVVC. They all had the diagnosis of a genital Candida infection by a physician at least four times during the previous year on the basis of history, clinical examination and at least one positive microscopic test for Candida morphotypes, in methylene-blue-stained smears prepared from posterior vaginal fornix samples. The patients presented with symptoms, such as vulvovaginal pruritis, irritation, unpleasant odor and dyspareunia, and/or with dysuria and burning at micturition. They had signs such as one or more of edema, erythema, fissures and caseous discharge.

The vaginal introitus was rubbed with a sterile, cotton-tipped swab moistened with physiological saline, which was plated on Sabouraud dextrose agar (Beckton Dickinson ${ }^{\circledR}$, Baltimore, USA) ${ }^{3}$ and chromogenic agar (CHROMagar ${ }^{\circledR}$, Paris) ${ }^{9}$, like swab samples from the posterior vaginal fornix. The order of the inoculation on the two media was changed for each consecutive case studied. Thereafter, vaginal lavage fluid was obtained by flushing the vagina with $3 \mathrm{ml}$ of sterile physiological saline and by regurgitating the fluid with the aid of a sterile syringe. Of the mixture, $0.1 \mathrm{ml}$ was inoculated on each of a Sabouraud and chromogenic agar plate.

Isolated strains of Candida were frozen at $-70^{\circ} \mathrm{C}$ in fetal calf serum for later speciation, which was done with the aid of API $32 \mathrm{C}^{\circledR}$ test kits and an automized identification system, Vitek ${ }^{\circledR}$ (BioMérieux, Marcy-l'Etoile, France). Chromogenic agar plates were read according to the criteria described by Pfaller and co-workers ${ }^{10}$.

The study was approved by the ethic committee of the Maternity Hospital. Written consents were obtained from the patients.

\section{RESULTS}

Table 1 shows the results of cultures made from swab samples from the introitus and posterior vaginal fornix and vaginal lavage samples, inoculated on Sabouraud and chromogenic agar. The recovery rate from the introitus was the same as that from the posterior vaginal fornix. The vaginal lavage samples missed two Candida-positive cases otherwise diagnosed by the swab samples.

C. albicans was the most frequent isolate, recovered in $40(87 \%)$ cases, followed by C. krusei detected in $4(9 \%)$ cases, C. glabrata in two and 
Table I Results of swab sample cultures from the introitus and the posterior vaginal fornix and of vaginal lavage samples from 91 patients with a history and current symptoms of recurrent vulvovaginal candidosis grown on Sabouraud and chromogenic agar

\begin{tabular}{llcc}
\hline & \multicolumn{2}{c}{$\begin{array}{c}\text { Number of positive Candida } \\
\text { cultures }\end{array}$} & \\
\cline { 2 - 3 } & $\begin{array}{c}\text { Sabouraud agar Chromogenic agar } \\
(n=91)\end{array}$ & $\begin{array}{c}(n=91) \\
\text { Sample type }\end{array}$ & p-value \\
\hline $\begin{array}{l}\text { Introital swab } \\
\begin{array}{l}\text { Posterior vaginal } \\
\text { fornix swab }\end{array}\end{array}$ & $43(47 \%)$ & $43(47 \%)$ & I \\
$\begin{array}{l}\text { Vaginal lavage } \\
\text { samples }\end{array}$ & $40(46 \%)$ & $43(47 \%)$ & I \\
\hline
\end{tabular}

C. parapsilosis in another case. There was only one case of mixed Candida infection, i.e. by C. albicans and C. krusei. The isolation rate on chromogenic agar was almost the same as on Sabouraud agar: the difference was $1.4 \%$ in favor of chromogenic agar.

A discrepancy in the result of speciation by means of chromogenic agar versus API 32C kits was only found in two of the 46 Candida-positive cases. In these two patients, C. albicans was identified by the API 32C kit, while C. glabrata was indicated to grow on the chromogenic agar. In another case, a mixed infection by C. albicans and C. krusei was found on the chromogenic agar, while only growth of C. albicans was identified by the API C32 and Vitek identification methods.

\section{DISCUSSION}

The diagnosis of genital Candida infections, in cases with a history of RVVC who attend with symptoms and signs suggestive of a new attack, fails more often than generally realized - even if both microscopic and culture studies are made. Thus the presence of Candida cannot be confirmed. Specialists in gynecology often misdiagnose the condition if not based on patient-close or central laboratory test results. In a previous study by Ledger ${ }^{12}$, the presence of Candida in the genital tract could only be confirmed in half of cases given the diagnoses of VVC or RVVC based on history, clinical examination and patient-close microscopy of vaginal secretion. In our study, half the women also proved to be culture-negative for Candida in spite of a 'typical' RVVC case history and findings at the clinical examination. In another study, Candida was identified by vaginal cultures in only $28 \%$ of 554 women with symptoms consistent with "candidal vaginitis' 13 . Thus, there are reasons to believe that a current genital Candida infection is commonly overdiagnosed in women diagnosed with RVVC. In fact, those women may suffer from other vaginal flora changes, such as those seen in bacterial vaginosis $(\mathrm{BV})^{14}$. Women may switch between $\mathrm{BV}$ and $\mathrm{VVC}^{15}$.

At least $10-15 \%$ of all women of reproductive age are colonized in the vagina by Candida, i.e. are carriers without any occurrence of either VVC or $\mathrm{RVVC}^{4}$. This colonization rate adds to the difficulty in diagnosing RVVC exclusively on the basis of genital yeast cultures, i.e. to differentiate carriers from cases with a true vaginal mucosal and/or introital invasion by yeast.

In our study, the sensitivity of chromogenic agar compared to Sabouraud dextrose agar for detection of Candida did not differ $(p=1)$. This is in accordance with the findings by Beighton and co-workers ${ }^{8}$. Nor did we find the use of vaginal lavage samples to positively influence the recovery rate of Candida.

The use of chromogenic agar has one disadvantage compared to Sabouraud agar, namely its rather high cost. On the other hand, chromogenic agar can identify most of the species occurring in the female genital tract. Consequently other expensive identification tests may be avoided. The use of an incubation temperature below $30^{\circ} \mathrm{C}$ does not allow speciation of Candida strains ${ }^{9,16}$ on chromogenic agar. Thus, chromogenic agar cannot be used at doctors' offices for speciation if no incubator is available.

C. albicans, C. krusei and C. tropicalis have been indicated to represent more than $99 \%$ of all yeast isolates from the human vagina ${ }^{7}$. In an Italian study of symptomatic women with a positive Candida culture, C. glabrata was the next most common species found, identified in 1207 of 3351 cases. C. krusei and C. tropicalis were found in 404 and 290 cases, respectively ${ }^{17}$. In our study, C. glabrata was found in only two cases.

The possibility of rapidly identifying the species of Candida by which a women is colonized 
increases the possibility of obtaining successful cure. Women infected with non-albicans species may not respond to azole drugs, to which such species are naturally resistant or have a low sensitivity $^{18}$. In that respect chromogenic agar serves a diagnostic purpose in RVVC cases.

\section{REFERENCES}

1. Bro F. The diagnosis of Candida vaginitis in general practice. Scand J Prim Health Care 1989;7:19-22

2. Emmerson J, Gunputrao A, Hawkswell J, et al. Sampling for vaginal candidosis: how good is it? Int J STD AIDS 1994;5:356-8

3. Nyirjesy P, Seeney SAM, Grody $\mathrm{MH}$, et al. Chronic fungal vaginitis: the value of cultures. $\mathrm{Am}$ J Obstet Gynecol 1995;173:820-3

4. Mendling W. Vulvovaginal Candidosis (Theory and Practice). Berlin: Springer-Verlag, 1988;12

5. Pfaller MA, Preston T, Bale M, et al. Comparison of the quantum II, API Yeast Ident, and automicrobic systems for identification of clinical yeast isolates. J Clin Microbiol 1988;26:2054-8

6. Patterson TF, Revankar SG, Kirkpatrick WR, et al. Simple method for detecting fluconazoleresistant yeast with chromogenic agar. J Clin Microbiol 1996;34:1794-7

7. Odds FC, Bernaerts R. CHROMagar candida, a new differential isolation medium for presumptive identification of clinically important candida species. J Clin Microbiol 1994;32:1923-9

8. Beighton D, Lundford R, Clark DT, et al. Use of CHROMagar Candida medium for isolation of yeasts from dental samples. J Clin Microbiol 1995;33: 3025-7

9. Koehler AP, Chu K-C, Houang ETS, Cheng AFB. Simple, reliable, and cost-effective yeast identification scheme for the clinical laboratory. J Clin Microbiol 1999;37:422-6

10. Pfaller MA, Houston A, Coffmann S. Application of CHROMagar Candida for rapid screening of clinical specimens for Candida albicans, Candida tropicalis, Candida krusei, and Candida (Torulopsis) glabrata. J Clin Microbiol 1996;34:58-61

RECEIVED 10/10/01; ACCEPTED 01/04/02
11. Embree JE, Lindsay D, Williams T, et al. Acceptability and usefulness of vaginal washes in premenarcheal girls as a diagnostic procedure for sexually transmitted diseases. Pediatr Infect Dis 1996; 15:662-7

12. Ledger WJ. Current problems in the diagnosis and treatment of candida vaginitis. Ital J Obstet Gynecol 1999;11:25-9

13. Eckert LO, Hawes SE, Stevens CE, et al. Vulvovaginal candidiasis: clinical manifestations, risk factors, management algorithm. Obstet Gynecol 1998;92:757-65

14. Zdolsek B, Hellberg D, Fröman G, et al. Vaginal microbiological flora and sexually transmitted diseases in women with recurrent and current vulvovaginal candidosis. Infection 1995;23:81-4

15. Mårdh $\mathrm{P}-\mathrm{A}$, Rodrigues $\mathrm{A}, \mathrm{Pina}-\mathrm{Vaz} \mathrm{C}$. Can in vitro observation explain interactions in vivo between a lactobacilli-dominated vaginal flora and vulvovaginal candidosis? Ital J Obstet Gynecol 2001;13: 89-93

16. Odds FC, Davidson A. 'Room temperature' use of CHROMagar Candida. Diagn Microbiol Infect Dis 2000;38:147-50

17. Parazzini F, Cintio Di E, Chiantera V, et al. Determinants of different candida species infections of the genital tract in women. Eur J Obstet Gynecol Reprod Biol 2000;93:141-5

18. Mårdh P-A, Rodrigues A, Genc M, et al. Facts and myths on recurrent vulvovaginal candidosis - a review on epidemiology, clinical manifestations, diagnosis, pathogenesis and therapy. Int $J$ STD $A I D S$, in press 


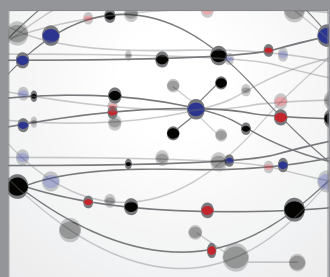

The Scientific World Journal
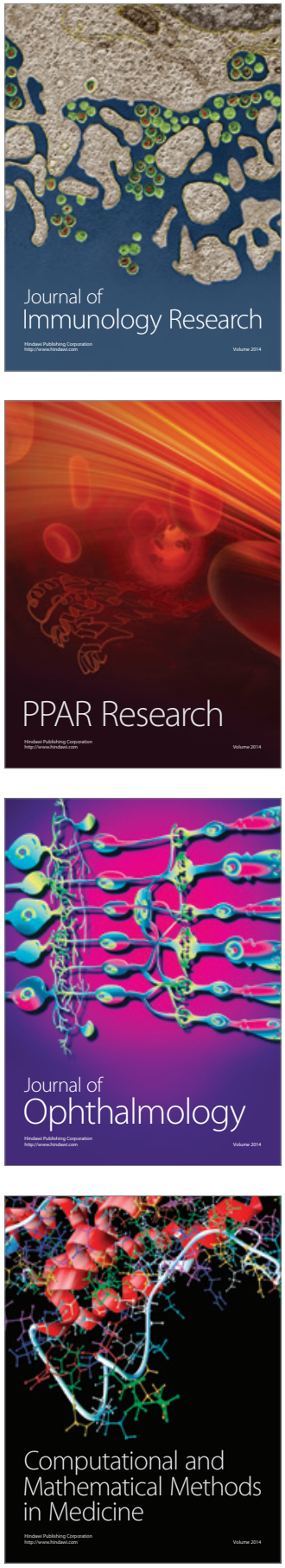

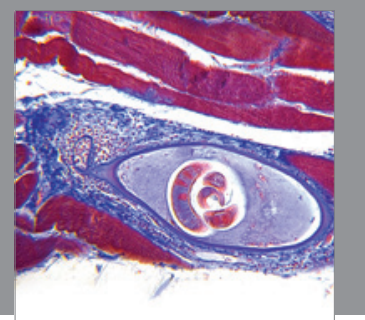

Gastroenterology

Research and Practice
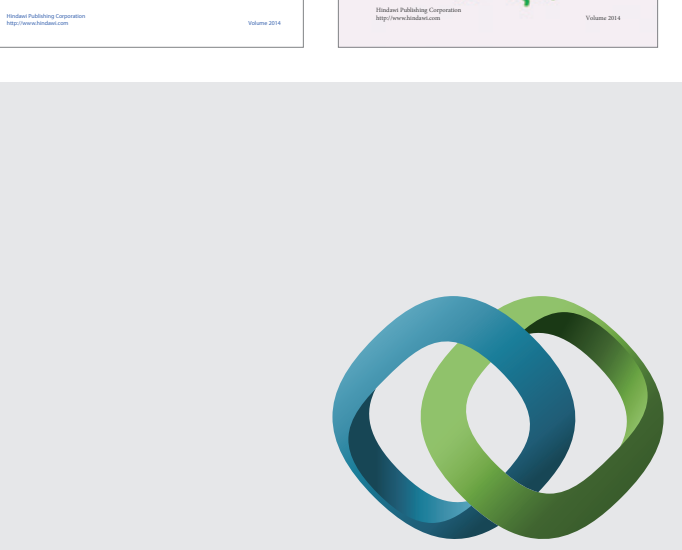

\section{Hindawi}

Submit your manuscripts at

http://www.hindawi.com
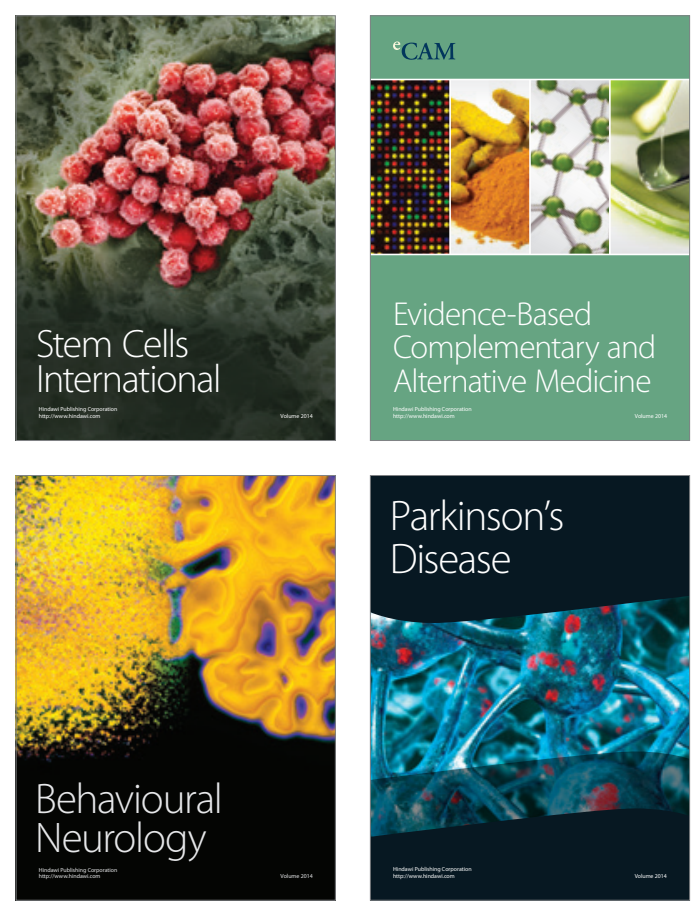

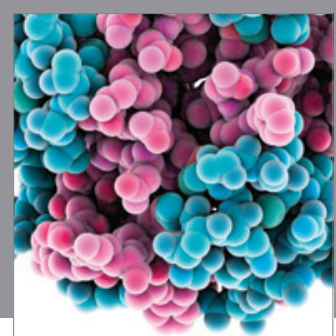

Journal of
Diabetes Research

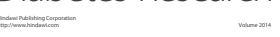

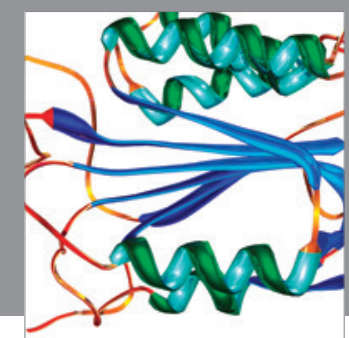

Disease Markers
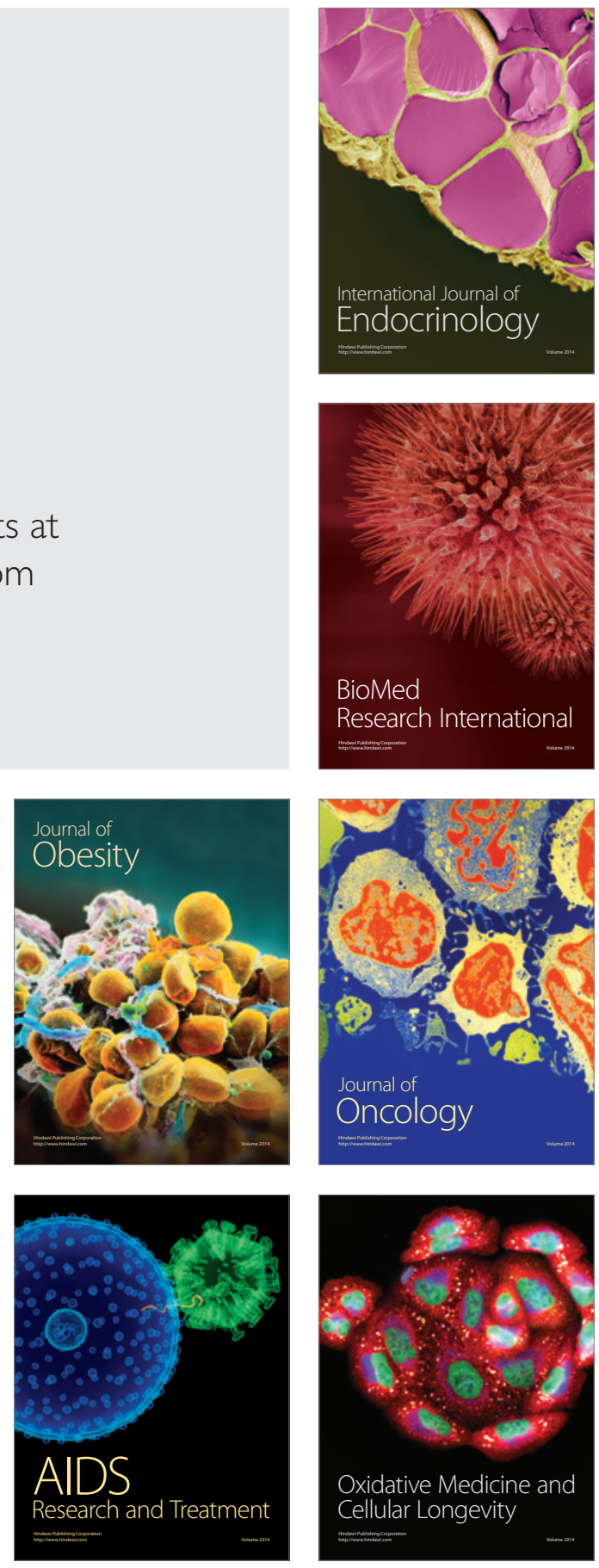